\title{
Correction to: Elicitation of Isatis tinctoria L. hairy root cultures by salicylic acid and methyl jasmonate for the enhanced production of pharmacologically active alkaloids and flavonoids
}

\author{
Published online: 9 April 2019 \\ C) Springer Nature B.V. 2019

\section{Correction to:} \\ Plant Cell, Tissue and Organ Culture (PCTOC) \\ (2019) 137:77-86 \\ https://doi.org/10.1007/s11240-018-01553-8
}

Qing-Yan Gai ${ }^{1}$. Jiao Jiao ${ }^{1}$ Xin Wang ${ }^{1} \cdot$ Yu-Ping Zang ${ }^{1} \cdot$ Li-Li Niu $^{1} \cdot$ Yu-Jie Fu ${ }^{1,2}$

In the original publication, only affiliation number 2 was indicated as the last author's affiliation. In fact, the author works for both affiliations.

Publisher's Note Springer Nature remains neutral with regard to jurisdictional claims in published maps and institutional affiliations.

Communicated by Wagner Campos Otoni.

Qing-Yan Gai and Jiao Jiao have contributed equally to this work.

The original article can be found online at https://doi.org/10.1007/s11240-018-01553-8.

Jiao Jiao

jj_nefu@163.com

$\triangle$ Yu-Jie Fu

yujie_fu@163.com

1 Key Laboratory of Forest Plant Ecology, Ministry of Education, Northeast Forestry University, Harbin 150040, People's Republic of China

2 Beijing Advanced Innovation Center for Tree Breeding by Molecular Design, Beijing Forestry University, Beijing 100083, People's Republic of China 